\title{
Exhaustion and emotional demands in China: a large-scale investigation across occupations
}

Kelly Z. Peng

Correspondence:

Kelly.z.peng@gmail.com

Department of Business

Administration, Hong Kong Shue

Yan University, Hong Kong, China

\begin{abstract}
As the Chinese economy moves toward a market-based model, employees are likely to face more emotional demands and exhaustion at work. However, there are some unique aspects to the emotional demands of work in the Chinese cultural context. We investigate emotional demands and exhaustion in China with a large-scale sample across the six major occupations identified by the Holland classification system. Results show that incumbents of social and enterprising jobs face higher emotional demands. Unexpectedly, exhaustion differs significantly between conventional and other types of jobs. Building on the Job Demand-Resources (JD-R) model, job crafting and the cultural context, we propose that the nonlinear relationship of emotional demands and exhaustion exists only when emotional intelligence is low. Our study may inform practitioners and policy makers in Chinese enterprises about emotional demands and exhaustion for various occupations and the importance of selection and training programs in emotional intelligence.
\end{abstract}

Keywords: Exhaustion, Burnout, Emotional demands, Emotional intelligence, JD-R model

\section{Introduction}

The Chinese economy is moving toward a more market-orientated model from a planning economy, and companies are working to improve services to both internal and external customers (Chow 2015). As a result, Chinese employees are likely to face more job demands in their work than ever before (Bakker and Demerouti 2007). According to the Job Demands-Resources (JD-R) model (Demerouti, Bakker, Nachreiner, and Schaufeli 2001; Bakker and Demerouti 2007), which is one of the most popular conceptual frameworks in studying employee burnout and exhaustion, the requirements for more prescribed emotions may be more likely to lead to exhaustion (Brotheridge and Grandey 2002; Grandey 2000; Morris and Feldman 1996; Zapf 2002), which is the core component of burnout. While this issue has been widely discussed in western societies (Schaufeli, Leiter, and Maslach 2009), it has gained attention in China only in the past decade (Jiang and Li 2004). Studies on Chinese employees' exhaustion are so far limited to occupations such as nursing and teaching (e.g., Whittington and Higgins 2002; Hao, Hong, Xu, Zhou, and Xie 2015; Zhang, Huang, and Guan 2014). Yet exhaustion is not limited to "people work" or "human service" occupations (e.g., Schaufeli and Maslach 1993; Schaufeli et al. 2009; Brotheridge and Grandey 2002) in market-based economies.

(c) The Author(s). 2017 Open Access This article is distributed under the terms of the Creative Commons Attribution 4.0 International License (http://creativecommons.org/licenses/by/4.0/), which permits unrestricted use, distribution, and reproduction in any medium, provided you give appropriate credit to the original author(s) and the source, provide a link to the Creative Commons license, and indicate if changes were made. 
China is also experiencing these trends, and a greater variety of jobs are now available that may be emotionally demanding, which in turn may lead to exhaustion according to the JD-R model. Empirically, however, we lack a complete understanding of the current situation on this issue, especially across various occupations.

At the same time, we are also interested in the unique pattern of emotional demands and exhaustion in China. Emotional expression is usually subject to specific cultural contexts (e.g., Law, Wong, and Song 2004). For example, Markus and Kitayama (1991) point out that people in independent-based western societies and interdependent-based Chinese society may display emotions in different ways. In China, social norms emphasize "harmony" within a certain social context or group while emotions experienced by individuals are minor and are either neglected or suppressed if they deviate from the group norm (Bockover 1995; Hansen 1992; Shweder and Bourne 1984). As a result, Chinese people tend to use dialectical ways of representing emotions rather than "show" and/or "represent" emotions obviously to oneself or in a social situation (Bagozzi, Wong, and Yi 1999; Wong, and Bagozzi 2005). Therefore, Chinese may have different patterns in perceiving emotional demands and/or exhaustion in various occupations. Specifically, will Chinese feel more demands if they are more socially pressured to display a particular type of emotion in a certain occupation? With the same logic, will Chinese experience more exhaustion if they are in occupations which have strict requirements for "display rules"? These are important research questions. However, we have limited knowledge of the specific pattern of experience of emotional demands and exhaustion among Chinese (Jiang and Li 2004), which requires a large-scale investigation that includes various occupations to explore the answer.

Furthermore, it is important to explore how exhaustion may be controlled among Chinese employees to help maintain employee wellbeing and improve productivity (Schaufeli and Enzmann 1998). According to the JD-R model (Demerouti, Bakker, Nachreiner, and Schaufeli 2001; Bakker and Demerouti 2007), job resources can help reduce the negative impact of emotional demands on exhaustion (e.g., Bakker, Demerouti, and Verbeke 2004). Considering that emotional intelligence is an important resource (Mayer, Caruso, and Salovey 1999) and has been investigated in the Chinese context (e.g., Law, Wong, Huang, and Li 2008; Peng, Wong, and Che 2010), we further examine whether and how exactly this resource can reduce the impact of emotional demands on exhaustion for Chinese employees. We will mainly build on the JD-R model and borrow from job drafting literature to investigate the possible relationships between the demands, resources, and exhaustion in Chinese culture, in which emotional expression is different from the West (Bagozzi, Wong, and Yi 1999; Wong, and Bagozzi 2005). This research may benefit Chinese companies by providing guidance for human resources practices in how to adopt the recommendations of the emotional intelligence literature to select and train employees on emotional intelligence when jobs have high emotional demands (e.g., Clarke 2006; Slaski and Cartwright 2003).

Therefore, I would like to contribute to the literature in the following three major ways: 1) By including six categories of occupation based on the Holland model and considering emotions in the Chinese cultural context, our research may provide more comprehensive empirical support to the basic arguments of the JD-R model, and explore how Chinese people experience and perceive emotional demands and exhaustion differently from westerners; 2) we further specifically include three typical types of 
social jobs in our investigation to enrich our empirical knowledge of the applicability of the JD-R model to social occupations in China; and 3) conceptually, our study also contributes to the JD-R model by incorporating job crafting in the Chinese context and examines the specific mechanism of the moderating role of emotional intelligence, in which both linear and nonlinear relationships are hypothesized.

\section{Exhaustion and emotional demands across occupations}

Exhaustion is thought to be the core component of burnout and in turn has received considerable attention (Leiter and Maslash 1988). Exhaustion refers to the depletion of emotional resources to the extent that employees feel they lack adaptive resources and cannot give any more to their jobs. Although exhaustion occurs in various occupations, professions that require more interpersonal interactions may be more likely to experience exhaustion (Leiter and Maslash 1988; Schaufeli and Maslach 1993). Past research has shown that workers in "social occupations" (e.g., social workers, sales, teachers and nurses) may have a higher chance of suffering from exhaustion (e.g., Cordes and Dougherty 1993; Liu, He, and Yu 2013). Although there are a few studies in China on social occupations (e.g., Whittington and Higgins 2002; Hao, et al. 2015; Zhang, et al. 2014), relatively little is known about exhaustion in other occupations.

The emotional demands of a job are an important reason for exhaustion (Hochschild 1983; Leiter and Maslash 1988; Schaufeli and Maslach 1993). To perform a job adequately, particularly a service-oriented job, employees may need to present particular types of emotion (Hochschild 1983; Grandey 2000). For example, bank tellers and flight attendants have to steadily present friendly and willingness-to-serve emotions; a funeral worker has to maintain sad or solemn emotions. These requirements are the emotional demands of an occupation (Brotheridge and Grandey 2002; Grandey 2000). Employees with jobs requiring frequent interactions with customers or other members of the organization are expected to express certain types of emotions according to prescribed "display rules" and thus face high emotional demands (Hochschild 1983; Morris and Feldman 1996; Schaubroeck and Jones 2000; Zapf 2002). In contrast, the emotional demands of auto mechanics are low; they spend most of their time at work dealing with machines and interact with customers infrequently. Thus, their jobs do not require them to present particular emotions according to "display rules". Thus, emotional demands vary across occupations (e.g., Wong, Wong, and Law 2005).

\section{The Holland model of occupational classification and emotional demands}

According to the Holland model, major occupations are classified into six types, namely, Realistic, Investigative, Artistic, Social, Enterprising, and Conventional. Social jobs involve performing tasks through interactions with people. Typical examples are nurses and social workers. Compared with other kinds of employment, social occupations described by Holland's model have high emotional demands as it involves primarily service sector jobs that require substantial prescribed "display rules" during intensive interpersonal interactions. Individuals in Realistic occupations, such as plumbers, bus drivers or machine operators, are required to work on concrete subjects such as machines or instruments and tools. Similarly, Conventional jobs like clerks or accountants involve tasks that are executed according to prescribed instructions, rules and regulations. People in these jobs follow instructions to perform their work without expressing emotion. 
On the contrary, Artistic jobs require incumbents to express feelings and emotions through various means such as singing, dancing or writing. Although they also need plenty of passion or emotion in their work, the emotion is likely to be inspired but not required. Artistic jobs rely on affective thinking, while Investigative and Enterprising jobs ask for rational thinking. Investigative jobs involve duties that mostly require abstract thinking, reasoning and logical deduction. Typical examples are scientists and computer programmers. Enterprising jobs involve mainly management functions and business deals. Typical examples are managers and entrepreneurs. Hence, among these jobs, individuals in social jobs experience the greatest emotional demands. Meanwhile, Chinese society is characterized as an interdependence-based culture and emphasizes "harmony" in social groups. More social interactions in social job may lead to more social pressure on job holders as to follow more intensive "display rules". As a whole, emotional demands may be experienced by social job holders, which are also evidenced among Chinese (Wong et al. 2005). Therefore, we hypothesize:

\section{Hypothesis $1 a$}

The emotional demands of social jobs are significantly higher than those of other kinds of jobs.

While it would still be informative to understand the overall situation of social occupation jobs as a whole, emotional demands and exhaustion may still differ for jobs within the social occupation category. It could help human resource practitioners within social occupations to know more about the relevant emotional demands and how much effort should be put into exhaustion prevention. In order to explore this possibility, we collected data from nurses, waiters and sales representatives within the social category. It is meaningful to know the difference between these three social jobs. Specifically, we expected that nurses may face higher emotional demands than waiters and sales representatives, because nurses need to handle the fear and suffering of patients and their families (e.g., Grandey, Foo, Groth, and Goodwin 2012), and therefore may face more negative emotions and conflicts. In such circumstances, nurses may feel more obliged to follow "display rules" while also facing more social pressure in Chinese social contexts. Waiters and sales representatives are serving healthy people with fewer emotional expressions and demands and therefore relatively fewer negative emotions and conflicts are confronted. Therefore, I hypothesize:

\section{Hypothesis 16}

The emotional demands of nurses are significantly higher than waiters and sales representatives.

According to the JD-R model, high emotional demands will lead to high exhaustion (Bakker et al. 2004). As social jobs are those with considerable emotional demands, it is reasonable to assume that incumbents are more likely to experience exhaustion. This should be valid for Chinese workers as well. Furthermore, as Chinese workers are embedded in an interdependent-based context, they are accustomed to a dialectical approach to dealing with emotions. When they need to follow "display rules" that are professionally required, they may feel even greater pressure and may be more likely to experience exhaustion in emotionally demanding occupations (Cheung and Tang 2007). I therefore hypothesize: 


\section{Hypothesis $2 a$}

Exhaustion is significantly higher for incumbents in social jobs than in other kinds of jobs.

For social occupations, nurses face more emotionally demanding situations than do waiters and sales representatives and thus may suffer more from exhaustion according to JD-R model. In both western and Chinese literature, nurses receive a lot of research attention because it is regarded as perhaps the best example of challenging "people work" in the burnout literature, which denotes a high percentage of incumbents suffering from exhaustion (e.g., Grandey et al. 2012). Thus we further hypothesize:

\section{Hypothesis $2 b$}

Exhaustion is significantly higher for nurses than waiters and sales representatives.

\section{The moderating role of emotional intelligence}

The basic argument of the JD-R model is that job demands will negatively affect employee wellbeing but resources can help incumbents to cope with the demands (Bakker et al. 2004). However, findings in previous studies conducted in the West on the moderating effect of job resources on job demands are inconsistent (Bakker et al. 2004; Bakker and Demerouti 2007). One possible reason may be that the JD-R model has ignored the possibility that incumbents may choose to craft their jobs (Wrzesniewski and Dutton 2001).

In job crafting, employees independently modify aspects of their jobs to improve the fit between the characteristics of the job and their own needs, abilities, and preferences (Berg, Dutton, and Wrzesniewski 2008). Grounded in the JD-R model, job crafters change either the level of job demands, the level of job resources, or both (Tims et al. 2012, 2013). Specifically, when there are insufficient resources to cope with the job demands, employees may attempt to redefine some of their tasks or the scope of their jobs in order to lower the level of job demands (Tims et al. 2013). For example, when a nurse is too tired to deal with patients, he/she may reduce his/her time dealing with each patient and may also reduce the quality of service to a lower level while still meeting the standards of requirements. For example, s/he could smile less and minimize communication with the patient to lower the emotional demands. Doing so may allow employees to maintain their energy levels and focus their efforts on their core work tasks which in turn will lower the level of exhaustion in the long run (Schaufeli et al. 2009; Tims et al. 2013).

One of the most important job resources that can help to cope with emotional demands discussed in the literature is emotional intelligence (Winkel, Wyland, Shaffer, and Clason 2011; Peng et al. 2010). Emotional intelligence refers to an employee's ability to handle emotional issues in various social contexts (Mayer and Salovey 1997; Law, Wong, and Song 2004; Peng, et al. 2010). Employees with higher emotional intelligence may be able to cope better with emotional demands and are less likely to suffer exhaustion (e.g., Peng et al. 2010). Specifically, job crafting is less likely to happen for employees with high emotional intelligence because they have sufficient resources to cope with the emotional demands of their job. On the contrary, job crafting is more likely to occur when the job holder has lower levels of emotional intelligence. When emotional demands are beyond their abilities to cope, they probably will craft their jobs in a way that helps match their capabilities and reduce their chances of exhaustion. 
Simply, they may craft the emotional demands to a lower level, such as minimizing smiling and communication with patients, more likely to happen with nurses with low emotional intelligence. As we know, emotional intelligence is an ability and may be hard to change within a certain period of time. Initially, emotional demands will increase the chance of exhaustion but only after reaching a certain level of emotional demands. Then, an increase in emotional demands will have the negative effect of exhaustion as employees no longer try to cope with the demands directly. Thus, we may observe a nonlinear relationship between emotional demands and exhaustion for employees with low emotional intelligence.

In the Chinese context, we believe that a moderated nonlinear relationship is more likely to occur. Chinese tend to use a dialectical way of representing emotions rather than using emotion to differentiate oneself from others or as a basis for social actions in an interdependent-based context (e.g., Bagozzi et al. 1999). That is, Chinese employees prefer to follow emotional demands to keep "harmony" in their workplaces. In this cultural context, incumbents with high emotional intelligence will continue to utilize their resources to cope with the demands. Certainly, employees with low emotional intelligence will also try to cope with the demands as far as possible. However, since they lack the required resources, they probably will engage in job crafting as an alternative to deal with the demands when they no longer can cope with the demands directly. In so doing, they are able to maintain their energy levels to perform their core work tasks, and in turn reduce exhaustion in the long run (Schaufeli et al. 2009; Tims et al. 2013). Therefore, I hypothesize:

\section{Hypothesis 3}

The relationship between emotional demands and exhaustion will be nonlinear when emotional intelligence is low. Specifically, emotional demands will have a positive impact on exhaustion, but after exceeding a particular level of emotional demands, its impact on exhaustion will become negative. The relationship will remain linear when emotional intelligence is high.

\section{Methodology}

\section{Sample and procedure}

A large-scale sample across the six major occupations identified by the Holland classification system was used in this study. The participants in this study were 703 employees in the following six types of occupations: manual workers (Realistic), researchers (Investigative), art designers at advertising companies (Artistic), middle-level managers (Enterprising), clerks in local government (Conventional), and hotel waiters/waitresses, sales clerks in shops, and hospital nurses (Social). Incumbents of the Realistic, Investigative, Enterprising, and Conventional occupations each came from a single organization. For the Social category, we selected three kinds of jobs, and incumbents of each job were employed by a single organization. For the Artistic category, incumbents were drawn from eight organizations because there were few incumbents of this job type within a single organization. The sample was collected in a middle-sized city in western China. The organizations were from major industries such as government, manufacturing, hotel, advertising, and information technology. The manufacturing company (manual workers, realistic occupation) and the construction company 
(managers, enterprising occupation), and provincial government (clerks, conventional occupation) are large organizations; others are medium-sized organizations. Detailed information is summarized in Table 1.

We interviewed a top-level manager from each organization to help us identify positions consistent with the Holland classification and asked for permission to distribute questionnaires to incumbents occupying those positions. A total of 1,200 questionnaires (with cover letters) were distributed on site by one of the authors. We explained in the cover letter that participation was voluntary and anonymous, and that the information collected would be used for research purposes only. Those willing to participate completed the questionnaires and submitted them to the author in person. This procedure helps protect the confidentiality of the data, and we believe incumbents who chose to participate provided honest responses. A total of 703 completed questionnaires were returned, representing a response rate of 58.5\%. Table 2 contains information on participant demographics.

\section{Measures}

Measure of emotional demands (ED)

We used the emotional demands scale for Chinese respondents developed by Wu (2003), which was based on scales published in previous studies (e.g., Brotheridge and Grandey 2002). The scale consists of 18 items that are statements of types of behavior commonly required in response to emotional demands. Respondents were asked to evaluate the frequency and effort respectively for each item on a seven-point scale. A sample item is "To perform my job, I have to speak nicely so that others will feel happy." The sum of frequency and effort scores indicates the level of emotional demands.

Measure of emotional intelligence (EI)

We used Wong's Emotional Intelligence Scale (WEIS), which was developed for Chinese respondents (Wong, Law, and Wong 2004). This 40-item scale is designed to capture the four ability dimensions of emotional intelligence, including: 1) Self Emotion Appraisal; 2) Other's Emotion Appraisal; 3) Use of emotions to facilitate performance; 4) Regulation of Emotion. In the first part, respondents are asked to choose the option that best reflects their likely reaction to each of 20 scenarios. An example scenario is "You have an important examination tomorrow and are studying hard in your room. Your family is watching a television program that you like very much. Since your house is small and the noise of the

Table 1 Information of the Sampled Occupations

\begin{tabular}{lll}
\hline Occupation type & Type of organization & No. of valid respondents \\
\hline Manual worker (Realistic) & State-owned manufacturing company & 97 \\
Researchers (Investigative) & Research center governed by central & 107 \\
& government & \\
Art designer (Artistic) & Privately owned advertising company & 75 \\
Middle-level managers (Enterprising) & State-owned large construction company & 104 \\
Clerks (Conventional) & Provincial government & 111 \\
Waiter/waitress & Joint venture hotel (four-star) & 98 \\
Sales Representative & Privately owned shopping mall & 96 \\
Nurse & Public hospital & 46 \\
(Social) & & \\
\hline
\end{tabular}


Table 2 Demographics of the Respondents

\begin{tabular}{lllc}
\hline Demographic variables & Categories & No. of respondents & Valid percentage \\
\hline Gender & Male & 327 & 46.5 \\
& Female & 300 & 42.7 \\
Missing data & 76 & 10.8 \\
Age (years) & $<20$ & 13 & 1.8 \\
& $21-30$ & 251 & 35.7 \\
& $31-40$ & 267 & 38.0 \\
& $41-50$ & 120 & 17.1 \\
Job tenure (years) & $51-60$ & 47 & 6.7 \\
& $\geq 61$ & 5 & 0.7 \\
& $>1$ & 44 & 6.3 \\
& $1-5$ & 207 & 29.4 \\
& $6-10$ & 160 & 22.8 \\
& $11-20$ & 174 & 24.8 \\
& $>20$ & 41 & 5.8 \\
Missing data & 77 & 11.0 \\
& SHigh school & 157 & 22.3 \\
Diploma & 177 & 35.2 \\
Bachelor & 267 & 38.0 \\
Master or above & 25 & 3.6 \\
Missing & 77 & 11.0 \\
\hline
\end{tabular}

television annoys you, you: (a) ask your family to turn off the television but videotape the program so that you and your family can watch it together tomorrow after your examination; (b) although a little bit uncomfortable, you put headphones on to reduce the noise so that you can concentrate on your studies."

The second part of WEIS contains 20 ability pairs. One item of each pair is related to emotional intelligence, and the other is related to other intelligence dimensions. Respondents are asked to choose one of the two abilities in each pair that best represents their strengths. An example of ability pairs is "ability to motivate oneself to face failure positively versus ability to learn to create an artistic object (e.g., pottery, painting)." Apart from the developmental samples, subsequent studies using Chinese samples have cross-validated the acceptable reliability and convergence, and discriminant and predictive validities of the scale (Wong, Wong, and Law 2007).

\section{Measure of exhaustion}

Exhaustion was measured by five items from the Maslach Burnout Inventory-General Survey, which were scored on a six-point scale (Schaufeli et al. 1996). An example item is "I feel exhausted after work." We directly used the Chinese version of the five items developed by Li and Shi (2003).

\section{Measure of control variables}

Demographics, including gender, age, job tenure, and education level were measured by multiple-choice items. These demographic factors are commonly included in the 
control variable list in the literature (e.g., Maslach et al. 2001). Occupation is assigned by researchers according to the job nature of respondents and the description in Holland's handbook. For example, manufacturing workers are classified as having a realistic occupation (as shown in Table 1). The detailed information about the job nature and responsibilities are collected from interviews with the top-level manager, who helped us to distribute and collect questionnaires in each company.

According to the literature on the JD-R model, social supports are also important resources to cope with emotional demands in the workplace (e.g., Baruch-Feldman, Brondolo, Ben-Dayan, and Schwarz 2002). We used the social support scale modified from Karasek's Job Content Survey (Karasek, Gordon, Pietrokovsky, Frese, Pieper, Schwartz, Fry, and Schirer 1985). We include supervisor and coworker supports as our control variables. The scale consists of five items for each type of support. Respondents were asked to evaluate the frequency of support they received from supervisors or coworkers on a seven-point scale. An example item is "My coworkers care about me."

\section{Analyses}

Three sets of analyses were conducted. First, confirmatory factor analysis (CFA) was conducted, and Harman's single-factor method (Podsakoff, Mackenzie, Lee, and Podsakoff 2003) was used to detect possible common method bias. For emotional intelligence, we followed the procedure of the original developer to create four indicators according to the four dimensions of EI (Mayer et al. 1999; Wong et al. 2004) because the response format for individual items is dichotomous. For the other constructs, we include all the items in the CFA analysis. The fitness of the one/two/ three-factor model was compared with that of the four-factor model. This helped determine the appropriateness of the measures used in the study. Second, emotional demands and exhaustion were compared among various occupations by One-way ANOVA and Pos-hoc test afterwards. Finally, the moderated nonlinear effect of occupations on the relationship between emotional demands and exhaustion was examined through hierarchical regression analyses.

\section{Results}

As expected from the JD-R model, emotional demands and emotional intelligence are significantly correlated with exhaustion $(r=0.31, p<0.01 ; r=-0.28, p<0.01$, respectively) as shown by Table 3 , which presents the descriptive statistics, reliabilities (coefficient

Table 3 Descriptive Statistics and Correlations among Measures

\begin{tabular}{|c|c|c|c|c|c|c|c|c|c|}
\hline & Mean (SD) & 1 & 2 & 3 & 4 & 5 & 6 & 7 & 8 \\
\hline 1. Gender & - & - & & & & & & & \\
\hline 2. Age & - & -0.07 & - & & & & & & \\
\hline 3. Tenure & - & -0.06 & $0.70^{* *}$ & - & & & & & \\
\hline 4. Education & - & $-0.11^{*}$ & 0.02 & $-0.11^{*}$ & - & & & & \\
\hline 5. SS & $3.80(0.63)$ & $0.11^{*}$ & -0.05 & -0.01 & 0.00 & $(0.85)$ & & & \\
\hline 6. El & $21.09(8.43)$ & 0.06 & 0.00 & -0.07 & 0.08 & $0.40^{* *}$ & $(0.76)$ & & \\
\hline 7. $E D$ & $127.23(21.07)$ & 0.05 & -0.07 & -0.06 & -0.02 & $0.33^{* *}$ & $0.16^{* *}$ & $(0.88)$ & \\
\hline 8. EX & $16.11(3.95)$ & -0.04 & 0.03 & 0.07 & -0.07 & $-0.21^{* *}$ & $-0.28^{* *}$ & $0.31^{* *}$ & $(0.78)$ \\
\hline
\end{tabular}


alphas), and correlations among all measures. All reliability coefficients were above .70 . CFA results indicate that the four-factor model (i.e., emotional demands, emotional intelligence, exhaustion and social supports) has an acceptable fit $\left(\chi^{2}=3270.66, d f=\right.$ $1424, p=0.00$; RMSEA $=0.047$; NNFI $=0.92$; IFI $=0.93$; and $\mathrm{CFI}=0.93$ ). We also tried different combinations of models with one, two and three factors. All of them show significant deterioration in fit when compared to the four-factor model. For example, the one-factor model is not acceptable $\left(\chi^{2}=5797.50, d f=1430, p=0.00\right.$; RMSEA $=0.084$; $\mathrm{NNFI}=0.82$; IFI $=0.83$; and CFI $=0.83$ ). The $\Delta \chi^{2}$ was 2526.84, $\Delta d f=6, p<0.001$.

Emotional demands and exhaustion were compared among the six occupations using one-way ANOVA. Results reported in Table 4 shows significant differences in emotional demands and exhaustion between the six occupations.

To further compare the scores of emotional demands and exhaustion among occupations, each pair of the six occupations was compared using post-hoc tests. For emotional demands, Table 5 show the results which indicate social occupations have significantly higher scores than all other occupations, thus Hypothesis 1a is supported. We also found enterprising occupation has significantly higher score than the other four occupations. For exhaustion, post-hoc comparison found that social occupations have not significantly higher scores than other occupations, thus Hypothesis $2 \mathrm{a}$ is not supported. Instead, we found conventional occupations have significantly lower score those other occupations except artistic one as shown in Table 6.

Among the three social jobs, as shown in Table 7, nurses have higher levels of emotional demands than waiters and salespersons. Hypothesis $1 \mathrm{~b}$ is supported. However, we found only exhaustion for sales is significantly lower than for waiters. Therefore, Hypothesis $2 b$ is not supported.

Hierarchical regression analysis was conducted to test Hypothesis 3. In step one of our regression analysis, all control variables were entered. In step two, square terms of emotional demands were entered. Table 8 shows that there is a nonlinear relationship between emotional demands and exhaustion $(\beta=-0.12, p<0.01)$. In step three, the interaction between emotional intelligence and the square term of emotional demands and required lower level variables were included. The product term was significant $\left(\beta=0.11, p<0.05 ; \Delta R^{2}=0.05, p<0.05\right)$. Following Aiken and West (1991), we plotted the interaction with values one standard deviation below and above the means of the moderator. The plot (see Fig. 1) shows that the nonlinear relationship between emotional demands and exhaustion appears only when emotional intelligence is low, and the relationship is linear when emotional intelligence is high. Thus, Hypothesis 3 is supported.

Table 4 Differences in Emotional Demands and Exhaustion among the Six Types of Occupations

\begin{tabular}{lcccc}
\hline Occupations & $E D($ Mean $)$ & $F$ value & EX (Mean) & $F$ value \\
\hline Social $(n=209)$ & 134.21 & $12.22^{* *}$ & 16.37 & $3.90^{* *}$ \\
Enterprising $(n=104)$ & 132.47 & & 16.44 \\
Conventional $(n=111)$ & 121.29 & & 14.80 \\
Investigative $(n=107)$ & 124.05 & 16.01 \\
Artistic $(n=75)$ & 118.35 & 15.81 \\
Realistic $(n=97)$ & 123.75 & 16.99 \\
\hline
\end{tabular}

${ }^{*}$ and ${ }^{* *}$ are significantly different from zero at the 5 and $1 \%$ level respectively; ED = emotional demands; EX = exhaustion 
Table 5 Post-hoc Comparison of Emotional Demands across the Six Occupation Types

\begin{tabular}{llc}
\hline & & Mean difference \\
\hline Social & Realistic & $10.46^{* *}$ \\
& Investigative & $10.16^{* *}$ \\
& Conventional & $12.92^{* *}$ \\
& Artistic & $15.86^{* *}$ \\
Enterprising & Realistic & $8.72^{* *}$ \\
& Investigative & $8.42^{* *}$ \\
& Conventional & $11.18^{* *}$ \\
& Artistic & $14.12^{* *}$ \\
\hline
\end{tabular}

* and ** are significantly different from zero at the 5 and $1 \%$ level respectively

\section{Discussion}

The present study is a relatively large-scale investigation conducted in China across six occupational types classified according to the Holland model. We found that social occupations face the highest emotional demands as expected. Thus, H1a is supported, which is consistent with the JD-R model as well. Enterprising occupations also scored relatively higher on emotional demands than other occupations except for social occupations. These findings expand our knowledge about emotional demands across occupations not addressed in the extant literature (Brotheridge and Grandey 2002). For exhaustion, H2a is not supported. Unexpectedly, we only found that exhaustion for conventional occupations is significantly lower than other ones. One possible reason for this finding may be due to the uniqueness of the cultural context. The dialectical expression of emotions, a feature of the Chinese context, incorporates both a high likelihood of co-occurrence of negative and positive emotions, and a greater ease when emotional complexity is experienced (Goetz, Spencer-Rodgers, and Peng 2008). That is, Chinese, although in an occupation with high emotional demands, which indicate high social pressure to perform "display rules", may be relatively accustomed to handle such emotional complexity, or even emotional dissonance. In the literature on exhaustion, emotional dissonance is the discrepancy between authentic and displayed emotions as part of the job (e.g., Heuven, and Bakker 2003; Grandey 2000; Rutter and Fielding 1988; Zapf 2002). The higher the emotional demands, the more emotional dissonance will occur. However, Chinese may be more likely to feel at ease to deal with the dissonance. Therefore, compared to low emotionally demanding occupations, people who are in occupations with high emotional demands may not suffer more emotional dissonance. In turn, the exhaustion experienced by people in occupations with high or low emotional demands may not be as

Table 6 Post-hoc Comparison of Exhaustion across the Six Occupation Types

\begin{tabular}{llc}
\hline & & Mean difference \\
\hline Conventional & Realistic & $-2.19^{* *}$ \\
& Investigative & $-1.21^{*}$ \\
& Artistic & -1.01 \\
Social & $-1.57^{* *}$ \\
Enterprising & $-1.64^{* *}$ \\
\hline
\end{tabular}

* and ${ }^{* *}$ are significantly different from zero at the 5 and $1 \%$ level respectively 
Table 7 Post-hoc Test of Emotional Demands and Exhaustion of Three Jobs in the Social Occupation Category

\begin{tabular}{lllc}
\hline \multicolumn{3}{l}{ Three types of social jobs } & Mean difference \\
\hline$E D$ & Nurse & Waiter/waitress & $20.62^{* *}$ \\
& & Sales & $16.32^{* *}$ \\
EX & Sales & Waiter/waitress & $-1.69^{* *}$ \\
& & Nurse & -1.58 \\
\hline
\end{tabular}

* and ** are significantly different from zero at the 5 and $1 \%$ level respectively; $E D=$ emotional demands; $E X=$ exhaustion

significantly differentiated as in Western societies. Another possible reason may be that they enjoy relatively high job security and stable compensation packages which may protect them from exhaustion. However, these government clerks experience emotional demands that are similar to those of other occupations, except for social and enterprising occupations, suggesting that even government employees now need to provide better service to internal and external customers. Future studies may further explore the possibilities.

To provide a more comprehensive picture of emotional demands and exhaustion in China, we also look into three specific social occupations: nurses, sales, waiters/waitress. Among the three social jobs, nurses have higher levels of emotional demands than waiters and salespersons. Hypothesis $1 \mathrm{~b}$ is supported. Unexpectedly, nurses' exhaustion does not significantly differ from the other two social jobs. Our results show that exhaustion in sales is significantly lower than for waiters. Thus, Hypothesis $2 \mathrm{~b}$ is not supported. As stated before, Chinese may be more likely to feel at ease in dealing with emotional dissonance. Thus, the exhaustion experienced by incumbents may have little difference for social occupations with different levels of emotional demands.

Table 8 Regression Analysis of Moderated Nonlinear Relationship between Emotional Demands and Emotional Intelligence on Exhaustion

\begin{tabular}{llll}
\hline & \multicolumn{2}{l}{ Dependent variable: $E X$} & \\
\cline { 2 - 4 } & Model 0 & Model 1 & Model 2 \\
\hline Control Variables & & & 0.22 \\
Occupation & $-0.10^{*}$ & 0.01 & -0.02 \\
Gender & -0.02 & -0.02 & -0.01 \\
Age & 0.01 & -0.01 & 0.08 \\
Tenure & 0.03 & 0.10 & -0.05 \\
Education & -0.04 & -0.07 & $-0.22^{* *}$ \\
Social Support & $-0.20^{* *}$ & $-0.30^{* *}$ & \\
Independent Variables & & & $0.44^{* *}$ \\
$E D$ & - & $0.44^{* *}$ & $-0.12^{* *}$ \\
$E D^{2}$ & - & $-0.12^{* *}$ & $-0.27^{* *}$ \\
$E I$ & - & - & -0.08 \\
$E D{ }^{*} E l$ & - & - & $0.11^{*}$ \\
$E D^{2} E l$ & - & - & $0.25^{* *}$ \\
$R^{2}$ & $0.04^{* *}$ & $0.21^{* *}$ & $0.05^{* *}$ \\
$\Delta R^{2}$ & - & $0.16^{* *}$ &
\end{tabular}




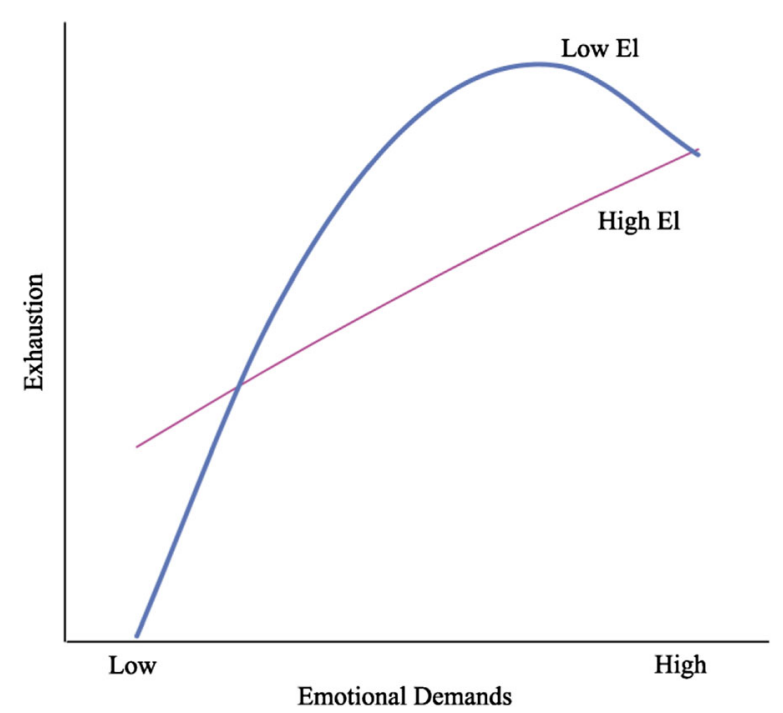

Fig. 1 Moderating Effect of Emotional Intelligence (El) on the Relationship between Emotional Demands and Exhaustion

Our results also support the moderating effect of emotional intelligence on the emotional demands-exhaustion relationship. However, it is more complicated than the interaction effect proposed by the JD- $R$ model when job crafting and the Chinese context are considered. For employees with high levels of emotional intelligence, a linear relationship is observed while for employees with low levels of emotional intelligence, the relationship is nonlinear. Specifically, low emotionally intelligent employees are more likely to have difficulties in dealing with emotional demands, but they will continue to try to do so in the Chinese context. They may try to craft their job to bring emotional demands to a lower level to match their capabilities and release tension which in turn will prevent exhaustion. This finding may be unique in the Chinese context as social norms emphasize complying with "display rules" in social contexts. With these findings, our study has several significant theoretical contributions and implications for human resource practice as follows.

\section{Theoretical contributions}

Theoretically, our paper contributes to the literature in three ways. First, the present large-scale investigation contributes to an understanding of emotional demands and exhaustion in China. It appears that as the Chinese economy moves toward a more market-based model, emotional demands and exhaustion are unavoidable although the Chinese cultural context has a unique impact. By understanding the cultural context, it may help us better understand how Chinese people will react to emotional demands and exhaustion. Future research may follow this direction and incorporate the specific business and cultural context in studying the phenomenon of emotional demands and exhaustion. The study further adds to the understanding that emotions have an important role in the workplace and that emotions need to be better understood and managed by organizations and employees (Li et al. 2014). 
Second, our study contributes to the JD-R model by incorporating an understanding of job crafting in the Chinese context and examines the moderating role of emotional intelligence on the emotional demands-exhaustion relationship. Past JD-R studies have found inconsistent results concerning the moderating role of resources on the demands-exhaustion relationship. By incorporating the possibility of job crafting, we are able to explain the specific moderating role of emotional intelligence. The different form the moderating effect takes when emotional intelligence is high or low expands our knowledge about the buffering effects of job resources in Chinese society. Future JD-R studies may follow this line of logic and continue to examine the specific moderating role of different types of resources on the demands-exhaustion relationship. This probably can help extend the applicability of the JD-R model in different culture contexts.

\section{Practical contributions}

Regarding practical contributions, the present large-scale investigation attempts to provide a more comprehensive picture of emotional demands and exhaustion across occupations in China. This may inform professional practitioners as to the characteristics of different occupations and differential risks of burnout. For example, organizations should provide more resources for the incumbents of social occupations to understand and deal with intense emotional demands to better perform their jobs, an area often neglected by traditional management in Chinese organizations. Moreover, we found that enterprising occupations also scored relatively high on emotional demands. This occupation has not previously been reported as having high emotional demands (Hochschild 1983; Brotheridge and Grandey 2002). This finding implies that organizations should provide appropriate external resources for these employees, which may have be neglected in the past. Our findings may also have reference value for policy makers in China, who need to re-examine other traditional demands in making industrial policies while developing health, safety and other labor regulations (Dunbar and Wasilewski 1985; Grandey, Foo, Groth, and Goodwin 2011). As China moves toward more legal protection of its labor force, protection from emotional demands should also be considered by legislators, labor union leaders, and government agencies.

The present study also compared three typical social occupations and found that the emotional demands of nurses are significantly higher than those of waiter/waitresses and sales clerks. Thus, even among typical service jobs, emotional demands appear to vary considerably. However, this result is not the same for exhaustion. It shows that the relationship between emotional demands and exhaustion within a social occupation may be more complicated than previously thought. These findings provide more detailed information to researchers and practitioners in China.

Our results indicate the importance of an employee's emotional intelligence in preventing exhaustion across various occupations. Organizations have some control over this factor through employee selection and training on emotional intelligence, which may improve both employee performance and long-term health (Grandey et al. 2011). Taken together, our findings help focus attention on the topic of emotional demands and exhaustion in China, especially in the increasingly important service industry. 


\section{Limitations and future research}

Two limitations of this study must be noted. First, as an initial effort to apply the JD-R model in the Chinese context, we examined only one type of job demand (emotional demands) and one type of job resource (emotional intelligence). Second, due to the nature of the constructs, we relied primarily on self-reported data, which may have resulted in inflated relationships due to common method variance. However, the key hypothesis of our study involves a moderated nonlinear relationship; therefore, our results should be much less susceptible to common method bias (Podsakoff et al. 2003). The CFA results above indicate that common method variance may be minimal. Future research could: 1) explore other types of job demands and resources to further refine and enrich the explanatory power of the model in the Chinese context; 2) use multiple indicators of different constructs, such as others' ratings of emotional intelligence (Law et al. 2004), and an objective indicator of burnout such as physiological symptoms of stress to avoid common method variance.

\section{Conclusion}

Through the lens of the unique Chinese culture of emotional expression, our research provides a more comprehensive picture on how Chinese people experience, perceive and react to emotional demands and exhaustion based on a large-scale sample $(n=703)$ across Holland's six major occupation types. By incorporating job crafting in the Chinese context, our research enriches the JD-R model in examining the specific moderating role of emotional intelligence.

Competing interests

The authors declare that they have no competing interests.

\section{Publisher's Note}

Springer Nature remains neutral with regard to jurisdictional claims in published maps and institutional affiliations.

Received: 9 September 2016 Accepted: 9 December 2016

Published online: 29 June 2017

\section{References}

Aiken, L. S., \& West, S. G. (1991). Multiple regression: Testing and interpreting interactions. Newbury Park: Sage.

Bagozzi, R. P., Wong, N., \& Yi, Y. (1999). The role of culture and gender in the relationship between positive and negative affect. Cognition and Emotion, 13(6), 641-672.

Bakker, A. B., \& Demerouti, E. (2007). The job demands-resources model: State of the art. Journal of Managerial Psychology, 22(3), 309-328.

Bakker, A. B., Demerouti, E., \& Verbeke, W. (2004). Using the job demands-resources model to predict burnout and performance. Human Resource Management, 43(1), 83-104.

Baruch-Feldman, C., Brondolo, E., Ben-Dayan, D., \& Schwarz, J. (2002). Sources of social support and burnout, job satisfaction, and productivity. Journal of Occupational Health Psychology, 7(1), 84-93.

Berg, J. M., Dutton, J. E., \& Wrzesniewski, A. (2008). What is job crafting and why does it matter. Ross School of Business, Regents of the University of Michigan. http://positiveorgs.bus.umich.edu/wp-content/uploads/What-is-Job-Craftingand-Why-Does-it-Matter1.pdf. Accessed August 2015.

Bockover, M. I. (1995). The concept of emotion revisited: A critical synthesis of Western and Confucian thought. In J. Marks \& R. T. Ames (Eds.), Emotions in Asian thought (pp. 161-180). New York: State University of New York Press.

Brotheridge, C. M., \& Grandey, A. A. (2002). Emotional labor and burnout: Comparing two perspectives on 'people work'. Journal of Vocational Behavior, 60, 17-39.

Cheung, F. Y. L., \& Tang, C. S. K. (2007). The influence of emotional dissonance and resources at work on job burnout among Chinese human service employees. International Journal of Stress Management, 14(1), $72-87$.

Chow, G. C. (2015). China's economic transformation (3rd ed.). Malden: Wiley-Blackwell.

Clarke, N. (2006). Emotional intelligence training: A case of caveat emptor. Human Resource Development Review, 5(4), 422-441.

Cordes, C. L., \& Dougherty, T. W. (1993). A review and an integration of research on job burnout. The Academy of Management Review, 18(4), 621-656.

Demerouti, E., Bakker, A. B., Nachreiner, F., \& Schaufeli, W. B. (2001). The job demands-resources model of burnout. Journal of Applied Psychology, 86(3), 499-512. 
Dunbar, R. L. M., \& Wasilewski, N. (1985). Regulating external threats in the cigarette industry. Administrative Science Quarterly, 30, 540-559.

Goetz, J., Spencer-Rodgers, J., \& Peng, K. (2008). Dialectical Emotions: How cultural epistemologies influence the experience and regulation of emotional complexity. In R. Sorrentino \& S. Yamguchi (Eds.), Handbook of motivation and cognition across cultures. Cambridge: Academic.

Grandey, A. A. (2000). Emotional regulation in the workplace: A new way to conceptualize emotional labor. Journal of Occupational Health Psychology, 5(1), 95-110.

Grandey, A., Foo, S. C., Groth, M., \& Goodwin, R. E. (2011). Free to be you and me: A climate of authenticity alleviates burnout from emotional labor. Journal of Occupational Health Psychology, 17(1), 1-14.

Grandey, A., Foo, S. C., Groth, M., \& Goodwin, R. E. (2012). Free to be you and me: a climate of authenticity alleviates burnout from emotional labor. Journal of Occupational Health Psychology, 17(1), 1-14.

Hansen, C. (1992). A Daoist theory of Chinese thought. New York: Oxford University Press.

Hao, S., Hong, W., Xu, H., Zhou, L., \& Xie, Z. (2015). Relationship between resilience, stress and burnout among civil servants in Beijing, China; Mediating and moderating effect analysis. Personality and Individual Differences, 83, 65-71.

Heuven, E. M., \& Bakker, A. B. (2003). Emotional dissonance and burnout among cabin attendants. European Journal of Work and Organizational Psychology, 12(1), 81-100.

Hochschild, A. R. (1983). The managed heart. Berkeley: University of California Press.

Jiang, X. J., \& Li, H. (2004). Service industry and China's economy: Correlation and potential of faster growth. Economic Research, 1, 4-15.

Karasek, R., Gordon, G., Pietrokovsky, C., Frese, M., Pieper, C., Schwartz, J., Fry, L., \& Schirer, D. (1985). Job content instrument: Questionnaire and user's guide. Los Angeles: University of Southern California.

Law, K. S., Wong, C. S., \& Song, L. (2004). Construct validity of emotional intelligence: Its potential utility of management studies. Journal of Applied Psychology, 89(3), 483-496.

Law, K. S., Wong, C. S., Huang, G., \& Li, X. (2008). The effects of emotional intelligence on job performance and life satisfaction for the research and development scientists in China. Asia Pacific Journal of Management, 25(1), 51-69.

Leiter, M. P., \& Maslash, C. (1988). The impact of interpersonal environment on burnout and organizational commitment. Journal of Organizational Behavior, 9(4), 297-308.

Li, C. P., \& Shi, K. (2003). The influence of distributive justice and procedural justice on job burnout. Acta Psychologica Sinica, 35(5), 677-684.

Li, Y., Ashkanasy, N. M., \& Ahlstrom, D. (2014). The rationality of emotions: A hybrid process model of decision-making under uncertainty. Asia Pacific Journal of Management, 31(1), 293-308.

Liu, J., He, Z., \& Yu, L. (2013). Meta-analysis of teachers' job burnout in China. In S. Li, Q. Jin, X. Jiang, \& J. J. J. H. Park (Eds.), Frontier and future development of information technology in medicine and education (pp. 1771-1778). Netherlands: Springer Science and Business Media.

Markus, H. R., \& Kitayama, S. (1991). Culture and the self: Implications for cognition, emotion, and motivation. Psychological Review, 98(2), 224.

Maslach, C., Schaufeli, W. B., \& Leiter, M. P. (2001). Job burnout. Annual Review of Psychology, 52(1), 397-422.

Mayer, J. D., \& Salovey, P. (1997). What is emotional intelligence? In P. Salovey \& D. Sluyter (Eds.), Emotional development and emotional intelligence: Implications for educators (pp. 3-31). New York: Basic Books.

Mayer, J. D., Caruso, D. R., \& Salovey, P. (1999). Emotional intelligence meets traditional standards for an intelligence. Intelligence, 27(4), 267-298.

Morris, J. A., \& Feldman, D. C. (1996). The dimensions, antecedents, and consequences of emotional labor. Academy of Management Review, 21(4), 986-1010.

Peng, K. Z., Wong, C. S., \& Che, H. S. (2010). The missing link between emotional demands and exhaustion. Journal of Managerial Psychology, 25(7), 777-798.

Podsakoff, P. M., Mackenzie, S. B., Lee, J. Y., \& Podsakoff, N. P. (2003). Common method biases in behavioral research: A critical review of the literature and recommended remedies. Journal of Applied Psychology, 88(5), 879-903.

Rutter, D. R., \& Fielding, P. J. (1988). Sources of occupational stress: An examination of British prison officers. Work and Stress, 2(4), 291-299.

Schaubroeck, J., \& Jones, J. R. (2000). Antecedents of workplace emotional labor dimensions and moderators of their effects on physical symptoms. Journal of Organizational Behavior, 21(2), 163-183.

Schaufeli, W. B., \& Enzmann, D. (1998). The burnout companion to study and practice. London: Taylor \& Francis.

Schaufeli, W. B., \& Maslach, C. (1993). Historical and conceptual development of burnout. In W. B. Schaufeli, C. Maslach, \& T. Marek (Eds.), Professional Burnout: Recent Developments in Theory and Research (pp. 1-16). Washington: Taylor \& Francis, DC

Schaufeli, W. B., Leiter, M. P., Maslach, C., \& Jackson, S. E. (1996). The Maslach Burnout Inventory-general survey. In C. Maslach, S. E. Jackson, \& M. P. Leiter (Eds.), Maslach Burnout Inventory (3rd ed.). Palo Alto: Consulting Psychologists Press.

Schaufeli, W. B., Leiter, M. P., \& Maslach, C. (2009). Burnout: 35 years of research and practice. Career Development International, 14(3), 204-220.

Shweder, R. A., \& Bourne, E. J. (1984). Does the concept of the person vary cross-culturally? In R. A. Shweder \& R. A. LeVine (Eds.), Culture theory: essays on mind, self, and emotion (pp. 158-199). Cambridge (England): Cambridge University Press.

Slaski, M., \& Cartwright, S. (2003). Emotional intelligence training and its implications for stress, health and performance. Stress and Health, 19(4), 233-239.

Tims, M., Bakker, A. B., \& Derks, D. (2012). Development and validation of the job crafting scale. Journal of Vocational Behavior, 80(1), 173-186.

Tims, M., Bakker, A. B., \& Derks, D. (2013). The impact of job crafting on job demands, job resources, and well-being. Journal of Occupational Health Psychology, 18(2), 230-240.

Whittington, R., \& Higgins, L. (2002). More than zero tolerance? Burnout and tolerance for patient aggression amongst mental health nurses in China and the UK. Acta Psychiatrica Scandinavica, 106(s412), 37-40.

Winkel, D. E., Wyland, R. L., Shaffer, M. A., \& Clason, P. (2011). A new perspective on psychological resources: Unanticipated consequences of impulsivity and emotional intelligence. Journal of Occupational and Organizational Psychology, 84(1), 78-94. 
Wong, N. Y., \& Bagozzi, R. P. (2005). Emotional intensity as a function of psychological distance and cultural orientation. Journal of Business Research, 58(4), 533-542.

Wong, C. S., Law, K. S., \& Wong, P. M. (2004). Development and validation of a forced choice emotional intelligence for Chinese respondents in Hong Kong. Asia Pacific Journal of Management, 21(4), 535-559.

Wong, C. S., Wong, P. M., \& Law, K. S. (2005). The interaction effect of emotional intelligence and emotional labor on job satisfaction: A test of Holland's classification of occupations. In C. E. J. Härtel, W. J. Zerbe, \& N. M. Ashkanasy (Eds.), Emotions in organizational behavior (pp. 235-250). Mahwah: Lawrence Erlbaum Associates.

Wong, C. S., Wong, P. M., \& Law, K. S. (2007). Evidence of the practical utility of Wong's emotional intelligence scale in Hong Kong and mainland China. Asia Pacific Journal of Management, 24(1), 43-60.

Wrzesniewski, A., \& Dutton, J. E. (2001). Crafting a job: Revisioning employees as active crafters of their work. Academy of Management Review, 26(2), 179-201.

Wu, C. Y. (2003). Emotional labor in work: Conceptual development, related constructs and psychological process. Taipei: Unpublished doctoral dissertation, National Taiwan University (in Chinese).

Zapf, D. (2002). Emotion work and psychological well-being: A review of the literature and some conceptual considerations. Human Resource Management Review, 12(2), 237-268.

Zhang, X. C., Huang, D. S., \& Guan, P. (2014). Job burnout among critical care nurses from 14 adult intensive care units in northeastern China: A cross-sectional survey. BMJ Open, 4, e004813. doi:10.1136/bmjopen-2014-004813.

Submit your manuscript to a SpringerOpen ${ }^{\circ}$ journal and benefit from:

- Convenient online submission

- Rigorous peer review

Open access: articles freely available online

- High visibility within the field

Retaining the copyright to your article

Submit your next manuscript at $\gg$ springeropen.com 